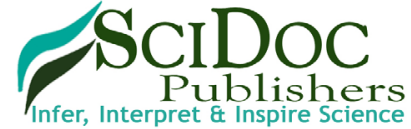

International Journal of Surgery and Research (IJSR)

ISSN 2379-156X

\title{
Evaluation of the Spectrum Of Co-Existing Injuries In Patients With Maxillofacial Trauma: A Retrospective Study
}

\author{
Research Article
}

Dr. Rajesh. $\mathrm{P}^{1}$, Dr. Vaishali. $\mathrm{V}^{2 *}$

${ }^{1}$ Professor and Head, Department of Oral and Maxillofacial Surgery, Chettinad Dental College and Research Institute, Kelambakkam, Chennai, India. ${ }^{2}$ Post Graduate, Department of Oral and Maxillofacial Surgery, Chettinad Dental College and Research Institute, Kelambakkam, Chennai, India.

\section{Abstract}

\begin{abstract}
Aim: To evaluate the types and frequency of co-existing injuries in patients sustaining maxillofacial trauma in a tertiary care centre of Tamil Nadu.

Settings: Trauma, being referred as silent epidemic of the era, potentially results in millions of death every year worldwide. The maxillofacial surgeon, being a part of the trauma team, gets to examine the patient first hand and thus has a shared responsibility to thoroughly assess the injuries incurred by other systems in addition to the survey of maxillofacial injuries. This study intends to highlight the need for an interdisciplinary management model in approaching facial trauma.

Methods: A retrospective analysis of 888 maxillofacial trauma cases reported to the emergency department during the last five years was done. Detailed review of primary and secondary survey was done. Age, gender, mechanism of injury, type and frequency of fracture, type and frequency of concomitant injury were recorded and subjected to statistical analysis.

Result: Associated injuries were sustained by 329 patients. Majority sustaining injuries were 20- 39 years and predominantly males. Soft tissue injury was frequently observed. Mandibular fractures were the most common among the hard tissue injury. Concomitant injuries observed in descending order was Neurologic, Orthopaedic, Ophthalmologic, Chest and finally abdominal.

Conclusion: Sound knowledge on the type and severity of associated injuries of other systems is mandatory for every surgeon to keep any latent life threatening conditions at the surgeons catch.
\end{abstract}

Keywords: Maxillofacial; Trauma; Concomitant Injuries; Traumatic Brain Injury; Oral Surgery.

\section{Introduction}

Patients with maxillofacial injuries are mostly seen to have co-existing injuries resulting from the trauma. The extent and degree of these injuries depend on the mechanism and impact of the trauma. The proficiency, with which a definite assessment is made, is a major factor in the prognosis of the patient [3]. Treatment of the maxillofacial injuries would be complex without a comprehensive perception of the damage incurred by other systems in the body of the victim [1]. There is increasing recognition that patients, who have sustained multiple injuries, benefit from early multidisciplinary management by a specialized unit, or trauma centre [2]. It is required of the maxillofacial surgeon attending such cases in the emergency department to promptly identify these concomitant injuries during the primary and secondary survey to avoid grave situations. This study intends to identify commonly occurring collateral injuries with maxillofacial trauma to render a directed patient care.

\section{Materials and Methods}

A retrospective analysis of a total of 6350 trauma patients, reported to the emergency department of our institution during the five year period, of March 2015 - March 2020, was carried out. Of these, a detailed review of 888 patients with maxillofacial trauma was done and data regarding age, gender, type of injury and its frequency, mechanism of injury, concomitant injuries of other organ systems and their frequencies was recorded. Patients with complete medical records along with radiologic records were included in the study. Maxillofacial injuries were recorded as soft

*Corresponding Author:

Dr. Vaishali. V, BDS,

Department of Oral and Maxillofacial surgery, Chettinad Dental College and Research Institute, Kelambakkam, Chennai- 603103, India.

Tel: 8056379290,8838017051

E-mail: vaish712.venkat@gmail.com

Received: December 19, 2021

Accepted: January 24, 2022

Published: January 25, 2022

Citation: Dr. Rajesh. P, Dr. Vaishali. V. Evaluation of the Spectrum Of Co-Existing Injuries In Patients With Maxillofacial Trauma: A Retrospective Study. Int J Surg Res. 2022;8(1):152 155. doi: http://dx.doi.org/10.19070/2379-156X-2200033

Copyright: Dr. Vaishali. $\mathbf{V}^{\odot}$ 2022. This is an open-access article distributed under the terms of the Creative Commons Attribution License, which permits unrestricted use, distribution and reproduction in any medium, provided the original author and source are credited. 
tissue injuries, fractures of the mandible, maxilla, zygomaticomaxillary complex, combined mandibular and middle third region and pan facial based on the clinical and radiological medical records. Concomitant injuries included are the neurological including traumatic brain injury, cervical spine injury, Orthopaedic, Ophthalmologic, Chest or pulmonologic, abdominal injuries. Records pertaining to the concomitant injuries were either taken from their emergency survey, or opinion obtained prior to the treatment for maxillofacial injury or treatment record attributing to the management of those specific system injuries. Cases with missing or incomplete medical records were excluded from the study. The data was tabulated and subjected to statistical analysis.

\section{Statistical Analysis}

SPSS software version 21 was used. Chi square test was applied and to assess the relationship between the variables Pearson's Correlation was used. In all the above probability value .05 is considered as significant level.

\section{Results}

A total of 888 maxillofacial trauma patients were identified for the study. $529(59.6 \%)$ of them belonged to 20-39 years of age, the maximum number to experience maxillofacial trauma followed by 40- 60 years with a total number of 194 (21.6\%), with $134(15.1 \%)$ less than 18 years and finally above 60 years with $31(3.5 \%)$ numbers. $673(75.8 \%)$ were males and $215(24 \%)$ females. There were a total of 343 hard tissue and 545 soft tissue maxillofacial injuries present. Mandibular fractures (148) were the most frequent, followed by zygomatico maxillary complex (77), combined mandibular-middle third (67), isolated maxillary (37) and pan facial fractures (17). Out of the 888 maxillofacial trauma patients, $329(37 \%)$ of them sustained associated injuries of other systems along with maxillofacial injuries. About $17.8 \%$ of them had traumatic brain injuries, $4.6 \%$ had cervical spine injuries, 7.4 $\%$ had orthopaedic injuries, $6.1 \%$ had ophthalmologic injuries, $1 \%$ had chest injuries and $0.1 \%$ had abdominal injuries (Table 1). Prevalence of different injuries with respect to hard and soft tissue maxillofacial injuries has been depicted in Figure 1. No statistical significance between the type of maxillofacial injury and prevalence of associated injuries was found. Frequency of occurrence of the concomitant injuries in different types of facial fractures was analysed and depicted in Table 2. Highest number of traumatic brain injuries were found with middle third fractures when compared to mandibular fractures, cervical spine injuries was highest in mandibular fractures, orthopaedic injuries in mandibular fractures and ophthalmologic injuries in middle third fractures.

\section{Discussion}

Nowadays, facial injuries have become a quotidian situation in the emergency rooms as face is highly vulnerable to trauma due to the fact that it is the most exposed region of our body [3]. It has been reported that about $50 \%$ of head and neck collateral injuries were observed in all trauma deaths [1]. This study aimed at illustrating the multisystem nature of the traumatic injuries associated with the fractures of facial skeleton. It is expected of the high energy forces causing facial fractures to have caused injuries to the other organ systems also. The extent and type of these injuries however depends on the mechanism of injury, force and its impact, patient factors like age, gender, co-existing morbidities etc. The diagnosis of such injuries has to be done on time and a coordinated interdisciplinary management protocol will have to be formulated in treating a poly trauma patient. While enormous

Figure 1. Frequency of prevalence of associated injuries in hard and soft tissue maxillofacial injuries.

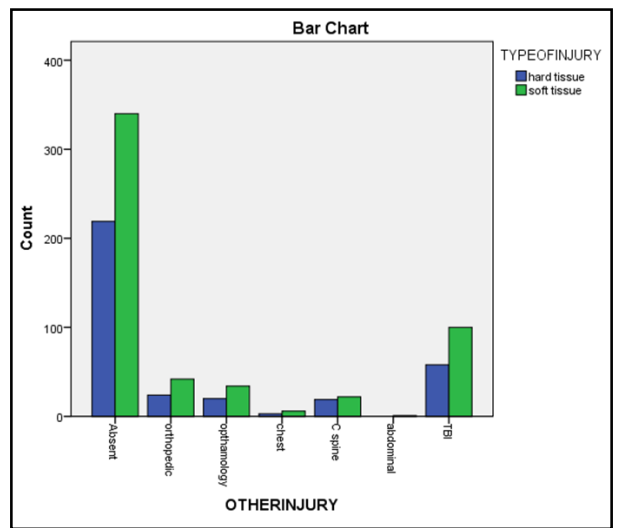

Table 1. Prevalence of associated injuries in maxillofacial trauma.

\begin{tabular}{|c|c|c|c|}
\hline \multicolumn{1}{|c|}{} & Frequency & Percent \\
\hline \multirow{7}{*}{} & Absent & 559 & 63 \\
\cline { 2 - 4 } & orthopedic & 66 & 7.4 \\
\cline { 2 - 4 } & opthamology & 54 & 6.1 \\
\cline { 2 - 4 } & chest & 9 & 1 \\
\cline { 2 - 4 } & C spine & 41 & 4.6 \\
\cline { 2 - 4 } & abdominal & 1 & 0.1 \\
\cline { 2 - 4 } & TBI & 158 & 17.8 \\
\cline { 2 - 4 } & Total & 888 & 100 \\
\hline
\end{tabular}


Table 2. Prevalence of concomitant injuries in various facial fractures.

\begin{tabular}{|c|c|c|c|c|c|c|c|c|}
\hline \multicolumn{2}{|c|}{} & \multicolumn{6}{|c|}{ FRACTURE } & \multirow{2}{*}{ Total } \\
\cline { 2 - 9 } & absent & mandible & combined & maxilla & zmc & panfacial & \\
\hline \multirow{4}{*}{$\begin{array}{c}\text { OTHER- } \\
\text { INJURY }\end{array}$} & Absent & 340 & 94 & 44 & 22 & 48 & 11 & 559 \\
\cline { 2 - 9 } & orthopedic & 42 & 13 & 5 & 0 & 5 & 1 & 66 \\
\cline { 2 - 9 } & opthamology & 34 & 9 & 2 & 4 & 2 & 3 & 54 \\
\cline { 2 - 9 } & chest & 6 & 1 & 1 & 0 & 1 & 0 & 9 \\
\cline { 2 - 9 } & C spine & 22 & 8 & 2 & 1 & 7 & 1 & 41 \\
\cline { 2 - 9 } & abdominal & 1 & 0 & 0 & 0 & 0 & 0 & 1 \\
\hline & TBI & 97 & 23 & 13 & 10 & 14 & 1 & 158 \\
\hline \multicolumn{2}{|c|}{ Total } & 542 & 148 & 67 & 37 & 77 & 17 & 888 \\
\hline
\end{tabular}

literature is available in assessing the existence of maxillofacial fracture in poly-trauma, scarce is the information validating the converse hypothesis. This study is designed with the aim of correlating the other injuries existing in maxillofacial trauma since maxillofacial surgeons form a part of the integrated trauma team that encounters the patient first hand after the injury. Thus it is imperative for every maxillofacial surgeon to be aware of the expected accessory injuries that has to be addressed during the primary and secondary survey.

In our study, the mean age group 31.40 of which 673 were males and 215 were females. No significant statistical correlation was found between the prevalence of concomitant injuries and, age and gender of the study population. Road traffic accident was the commonest cause followed by interpersonal violence and the others. This was in accordance with the study by Deliverska et al, Follmar et al [5], where they discussed possible random pattern of mechanical trauma, with forces distributed to the entire body that is conducive to injury to multiple parts of the body. With this opinion, on correlating the existence of associated injuries to the mechanism of trauma, Road traffic accident proves to cause poly trauma than the assault since the latter is directed to a particular portion of the body. . In our study most common fracture was mandibular (16\%), followed by ZMC (8.6\%), combined mandibular and middle third (7.5\%), isolated maxillary $(4.16 \%)$, pan facial $(1.9 \%)$, soft tissue injury (61\%). Among the associated injuries, in the descending order is the neurological including Traumatic Head Injury and Cervical spine injury, followed by Orthopaedic, ophthalmologic, pulmonologic and abdominal injuries.

Frequently occurring concomitant injury in our study was Traumatic Head Injury $(17.8 \%)$ which varied between mild, moderate and severe injuries. Maxillofacial injuries comprising hard tissue as well as soft tissue injuries can be associated with traumatic brain injuries due to the impact of forces transmitted through the head and neck [8]. Davidoff et al found a strong association of traumatic head injuries with facial fractures while Haug et al reported $76 \%$ incidence of traumatic brain injuries with maxillofacial fractures. In our study $46.7 \%$ of 124 cases of facial fractures presented with traumatic brain injuries. Higher prevalence rate could be related with middle face injuries owing to the complexity of anatomy with lesser density of the bones and its proximity to the skull. $4.6 \%$ of the sample sustained cervical spine injuries along with maxillofacial fractures. Hackl et al reported a rate of $19.2 \%$ of prevalence of cervical spine injuries in their study while several authors have reported a prevalence rate ranging between 0 to
$4.3 \%$ [4]. Generally a maxillofacial trauma patient is assumed to have sustained cervical spine injuries unless proven otherwise. If the neurologic status of the patient with respect to injury of brain and cervical spine could not be discretely ruled out, it is mandatory to go for radiologic imaging prior to maxillofacial management protocol. All the procedures should be deferred until the patient is found neurologically stable. The pattern and severity of these injuries highly influences the management protocol and hence should be ruled out with utmost importance.

Neurologic injuries are succeeded by orthopaedic injuries with $7.4 \%$ of total coexisting injuries. Deliverske et al reported $22 \%$ of orthopeadic injuries in patients with maxillofacial fractures which supports the results of our study that had $29.7 \%$ of the total facial fractures. This could be attributed again to multisystem involvement of a serious trauma and its impact. Often these injuries do not contra indicate timely management of maxillofacial injuries and if needed, interdisciplinary simultaneous surgical management has also been opted by the surgeons. But such decisions purely depends on the type of procedures, duration of surgery, general status of the patients, anticipated complications like intraoperative blood loss or hypothermia and fitness of the patient for anaesthetic procedures.

Ophthalmologic injuries form a significant proportion of this lot $(6.1 \%)$. These occur often as a complication of upper and middle third fractures especially orbito- zygomatico- maxillary complex fractures. Precise evaluation of changes in ocular structure and function is of prime importance to alleviate the risk of morbidity in the future. Pulmonologic injuries form of the total injuries. Though relatively lower in number than the others stated, missed diagnosis of the chest injuries often puts the patient's life at risk. Pulmonologic injuries can vary from fracture of the sternum or rib to injury to the tissues of the lungs. Chest $\mathrm{x}$-ray plays an inevitable role in the preliminary assessment of a poly trauma patient and if required higher imaging modality like CT or MRI can be preferred. Often these injuries are associated with severely injured trauma patients. Abdominal injuries are insignificant in values but do occur in rare cases. But one should be cautious and be aware of such occurrences so as to combat such unforeseen emergencies if occurs.

Except for the neurological status, other injuries don't cause significant delay in the fractures of the facial skeleton. The results of the study highlights the relatively high prevalence of collateral injuries proves to be a reminder of acuity of these patients and 
need for a multidisciplinary approach to the trauma for rendering directed comprehensive care in whole, volume after initial stabilization of the patient. Precautious examination and identification is expected of the surgeon. Most importantly, fractures from road traffic accident should never be imagined as an isolated injury but a part of spectrum of significant critical injury requiring system by system assessment. Based on the results of the present study, coexisting injuries in areas other than the face in poly trauma patients should be expected first and foremost as trauma that involves sufficient energy to fracture the bones of the facial skeleton is also likely to distribute a substantial amount of force to other parts of the body, and thus cause injury.

\section{Conclusion}

Knowledge of the frequency of concomitant injuries existing with the maxillofacial injuries post trauma serves as guidance to prompt identification and comprehensive management of the patient. Also it emphasises not to narrow down the focus on maxillofacial region but to widen the thoroughness of the assessment to the other systems that could potentially lead to catastrophic consequences if neglected.

\section{References}

[1]. Scherbaum Eidt JM, De Conto F, De Bortoli MM, Engelmann JL, Rocha FD. Associated injuries in patients with maxillofacial trauma at the hospital sáo vicente de paulo, passo fundo, Brazil. J Oral Maxillofac Res. $2013 \mathrm{Oct}$ 1;4(3):e1.Pubmed PMID: 24422034.

[2]. Down KE, Boot DA, Gorman DF. Maxillofacial and associated injuries in severely traumatized patients: implications of a regional survey. Int J Oral Maxillofac Surg. 1995 Dec;24(6):409-12.Pubmed PMID: 8636636.

[3]. Patil SG, Munnangi A, Joshi U, Thakur N, Allurkar S, Patil BS. Associated Injuries in Maxillofacial Trauma: A Study in a Tertiary Hospital in South India. J Maxillofac Oral Surg. 2018 Dec;17(4):410-416.Pubmed PMID: 30344378 .
[4]. Roccia F, Cassarino E, Boccaletti R, Stura G. Cervical spine fractures associated with maxillofacial trauma: an 11-year review. J Craniofac Surg . 2007 Nov 1;18(6):1259-63.

[5]. Follmar KE, DeBruijn M, Baccarani A, Bruno AD, Mukundan S, Erdmann $\mathrm{D}$, et al. Concomitant injuries in patients with panfacial fractures. J Trauma Acute Care Surg. 2007 Oct 1;63(4):831-5.

[6]. Alvi A, Doherty T, Lewen G. Facial fractures and concomitant injuries in trauma patients. Laryngoscope. 2003 Jan;113(1):102-6.

[7]. Carlin CB, Ruff G, Mansfeld CP, Clinton MS. Facial fractures and related injuries: a ten-year retrospective analysis. J Craniomaxillofac Trauma. 1998 Summer;4(2):44-8.Pubmed PMID: 11951431.

[8]. Rajandram RK, Syed Omar SN, Rashdi MF, Abdul Jabar MN. Maxillofacial injuries and traumatic brain injury--a pilot study. Dent Traumatol. 2014 Apr;30(2):128-32.Pubmed PMID: 23782407.

[9]. Davidoff G, Jakubowski M, Thomas D, Alpert M. The spectrum of closedhead injuries in facial trauma victims: incidence and impact. Ann Emerg Med. 1988 Jan;17(1):6-9.Pubmed PMID: 3337417.

[10]. Ajike SO, Adebayo ET, Amanyiewe EU, Ononiwu CN. An epidemiologic survey of maxillofacial fractures and concomitant injuries in Kaduna, Nigeria. Niger J Surg Res. 2005;7(3):251-5.

[11]. Obuekwe ON, Etetafia M. Associated injuries in patients with maxillofacial trauma. Analysis of 312 consecutive cases due to road traffic accidents. J Med Biomed Res. 2004 Jun;3(1):30-6.

[12]. McGoldrick DM, Fragoso-Iñiguez M, Lawrence T, McMillan K. Maxillofacial injuries in patients with major trauma. Br J Oral Maxillofac Surg. 2018 Jul 1;56(6):496-500.

[13]. Singaram M, G SV, Udhayakumar RK. Prevalence, pattern, etiology, and management of maxillofacial trauma in a developing country: a retrospective study. J Korean Assoc Oral Maxillofac Surg. 2016 Aug;42(4):174-81. Pubmed PMID: 27595083.

[14]. Fama F, Cicciu M, Sindoni A, Nastro-Siniscalchi E, Falzea R, Cervino G, et al. Maxillofacial and concomitant serious injuries: An eight-year single center experience. Chin J Traumatol. 2017 Feb;20(1):4-8.Pubmed PMID: 28209449.

[15]. Pungrasmi P, Haetanurak S. Incidence and etiology of maxillofacial trauma: a retrospective analysis of King Chulalongkorn Memorial Hospital in the past decade. Asian Biomed. 2017 Aug 1;11(4):353-8.

[16]. Reich W, Surov A, Eckert AW. Maxillofacial trauma - Underestimation of cervical spine injury. J Craniomaxillofac Surg. 2016 Sep;44(9):1469-78. Pubmed PMID: 27527678.

[17]. Choonthar MM, Raghothaman A, Prasad R, Pradeep S, Pandya K. Head injury-a maxillofacial surgeon's perspective. J Clin Diagn Res. 2016 Jan;10(1):ZE01. 